\title{
Predictive value of the ratio of 8-hydroxydeoxyguanosine levels between cancerous and normal tissues in patients with stage II/III colorectal cancer
}

\author{
HIROSHI KITAGAWA ${ }^{1}$, YOSHIHIKO KITAJIMA ${ }^{1,2}$, KEITA KAI $^{3}$, SHO KOMUKAI $^{4}$, \\ TOMOKAZU TANAKA $^{1}$, YASUO KOGA ${ }^{1}$, TATSUYA MANABE ${ }^{1}$ and HIROKAZU NOSHIRO ${ }^{1}$ \\ ${ }^{1}$ Department of Surgery, Saga University Faculty of Medicine, Saga $849-8501 ;{ }^{2}$ Department of Surgery, \\ NHO Higashisaga Hospital, Miyaki-Cho, Miyaki-Gun, Saga 849-0101; ${ }^{3}$ Department of Pathology, \\ Saga University Hospital, Saga 849-8501; ${ }^{4}$ Division of Biomedical Statistics, \\ Department of Integrated Medicine, Graduate School of Medicine, \\ Osaka University, Suita-City, Osaka 565-0871, Japan
}

Received December 12, 2018; Accepted March 13, 2019

DOI: $10.3892 /$ or.2019.7086

\begin{abstract}
Reactive oxygen species (ROS) accumulation is known to induce carcinogenesis and accelerate cancer progression. 8 -Hydroxydeoxyguanosine $(8-\mathrm{OHdG})$ is a specific marker of ROS-mediated DNA damage. Therefore, we analysed 8-OHdG levels in cancerous and normal tissue DNA via enzyme-linked immunosorbent assay (ELISA) using 97 tissue specimens obtained from surgically-treated patients with stage II/III colorectal cancer (CRC). Additionally, 8-OHdG levels in these tissues were also assessed via quantitative immunohistochemistry (qIHC). To eliminate individual background variables, the ratio of $8-\mathrm{OHdG}$ levels between cancerous and normal tissues was calculated using both techniques. A comparative analysis demonstrated that the 8-OHdG ratio in DNA was significantly correlated with both lymph node metastasis and lymphatic invasion. Multivariate analysis revealed that a high 8 -OHdG ratio in DNA was independently correlated with poor prognosis. These results suggest that the $8-\mathrm{OHdG}$ ratio in DNA reflects ROS-induced cancer progression. Conversely, a low 8-OHdG ratio as estimated via qIHC was an independent factor for poor prognosis. In Kaplan-Meier analysis, the combination of a high 8-OHdG ratio in DNA (ELISA) and a low 8-OHdG ratio in cytoplasm (qIHC) was associated with markedly worse patient prognosis than other combinations. Combined evaluation of the $8-\mathrm{OHdG}$ ratio using ELISA and qIHC may
\end{abstract}

Correspondence to: Dr Yoshihiko Kitajima, Department of Surgery, Saga University Faculty of Medicine, 5-1-1 Nabeshima, Saga 849-8501, Japan

E-mail: kitajima.yoshihiko.eg@mail.hosp.go.jp

Key words: colorectal cancer, 8-hydroxydeoxyguanosine, enzymelinked immunosorbent assay, quantitative immunohistochemistry, reactive oxygen species be pivotal for predicting surgical outcomes for patients with stage II/III CRC.

\section{Introduction}

Colorectal cancer (CRC) is one of the principal causes of cancer-related deaths worldwide (1). Reactive oxygen species (ROS) are believed to substantially influence carcinogenesis and cancer progression in solid tumors (2-5). ROS are generated intracellularly as by-products of the electron transport chain in mitochondria (3). In solid tumors, including CRC, ROS generation in mitochondria is increased under several conditions such as tumor hypoxia (6-8). Alternatively, inflammation and exposure to certain foods and tobacco are important aetiological factors of sporadic CRC $(9,10)$. These factors extracellularly increase ROS formation in the intestinal mucosa $(9,10)$. When ROS overwhelm the cellular antioxidant defence system, oxidative stress occurs (11). Oxidative stress causes ROS-mediated damage in DNA in epithelial cells, thereby triggering the appearance of genetic mutations and leading to carcinogenesis $(2,12)$. Furthermore, several studies have reported that ROS accumulation also increases the malignant characteristics of cancer cells, such as invasion, angiogenesis and metastasis, by activating several signalling molecules $(2,4,13,14)$. Therefore, assessing ROS levels in CRC tissues may contribute to the assessment of malignant potential in individual patients. However, directly measuring ROS is challenging due to their short lifespan (15). For instance, it has been reported that the lifespan of hydroxyl radical, the most harmful ROS, is $<1 \mathrm{~ns}(15)$.

8-Hydroxydeoxyguanosine $(8-\mathrm{OHdG})$ is a specific marker of 2'-deoxyguanosine damage in DNA after an ROS-mediated attack. In general, 8-OHdG in nuclear DNA can be formed by the incorporation of $8-\mathrm{OHdGTP}$ or by direct oxidation of DNA guanine bases (16). 8-OHdG accumulation in DNA increases the occurrence of $\mathrm{G}: \mathrm{C}$ to $\mathrm{T}: \mathrm{A}$ transversions, and these mutations are believed to initiate carcinogenesis (16). To date, $8-\mathrm{OHdG}$ has been one of the most widely used 
oxidative stress biomarkers. Recently, a specific antibody for 8-OHdG was developed, thereby permitting measurements of 8-OHdG levels in serum, urine and cancer tissues via immunohistochemistry (IHC) or enzyme-linked immunosorbent assay (ELISA) (17). However, few studies have investigated whether 8-OHdG accumulation in CRC tissues is correlated with CRC progression.

In the present study, using 97 pairs of CRC and matched normal tissues, 8-OHdG levels in DNA were analysed by ELISA, whereas its levels in the cytoplasm were investigated by quantitative IHC (qIHC). Furthermore, we estimated the ratio of $8-\mathrm{OHdG}$ levels between cancerous and normal tissues using both ELISA and qIHC and examined the correlation of these 8-OHdG ratios with several clinicopathological factors and patient survival. The clinical application of the $8-\mathrm{OHdG}$ ratio as a prognostic factor in patients with CRC was evaluated.

\section{Materials and methods}

Standard protocol approvals and patient consent. All participants provided written informed consent, and this research protocol was approved by the Saga University Institutional Review Board (2018-01-R-04). All normal and cancerous colorectal tissue samples were obtained during surgery according to the guidelines of the Human Tissue Research Committee at our hospital.

Patients and tissue collection. Between January 2003 and December 2011, 102 pairs of cancerous and normal tissue samples were collected from consecutively recruited patients who underwent curative surgery for pathologically confirmed stage II or III primary CRC at Saga University Hospital in Japan. All patients (stage II, $n=56$; stage III, n=46) were registered in our prospectively maintained comprehensive database, which contained their medical records including surgical outcomes. Specimens from cancerous and normal regions in the resected tissues were immediately frozen using liquid nitrogen and stored at $-80^{\circ} \mathrm{C}$ until use. Thirty-one patients with a good performance status received adjuvant chemotherapy following surgery due to the presence of lymph node metastasis or lymphatic invasion.

Quantitative measurement of 8-OHdG levels in tissue DNA by ELISA. Before extracting DNA, we homogenised tissue specimens on ice. DNA was extracted from the homogenates using a DNA Extractor ${ }^{\circledR}$ TIS Kit (Wako Pure Chemical Industries, Ltd., Osaka, Japan). Obtained DNA was digested into mononucleosides via treatment with nuclease P1 followed by alkaline phosphatase using an $8-\mathrm{OHdG}$ Assay Preparation Reagent Set (Wako Pure Chemical Industries, Ltd.). 8-OHdG levels were assessed in tissues using a commercially available highly sensitive ELISA kit for 8-OHdG (cat. no. KOG-HS10/E; Japan Institute for the Control of Aging, Fukuroi, Shizuoka, Japan) according to the manufacturer's instructions. In brief, 96-well plates were pre-coated with the 8 -OHdG antigen. Both a DNA solution and monoclonal antibody against 8-OHdG were added to 96-well plates, which were incubated at $4^{\circ} \mathrm{C}$ overnight. During this step, the monoclonal antibody reacted competitively with $8-\mathrm{OHdG}$ in sample solutions and $8-\mathrm{OHdG}$ bound to the plate. After washing the plate with PBS, an enzyme-labelled secondary antibody was added, followed by incubation at room temperature for $1 \mathrm{~h}$. Next, unbound enzyme-labelled secondary antibody was removed by washing the plate. Finally, the plate was incubated with $100 \mu \mathrm{l}$ of the reaction-terminating solution for $15 \mathrm{~min}$ in the dark, and colour development was measured on a plate reader at $450 \mathrm{~nm}$.

IHC analysis of 8-OHdG expression. For IHC, paraffin blocks $(20 \mathrm{~mm} \times 30 \mathrm{~mm})$ were first sectioned on slides at a thickness of $4 \mu \mathrm{m}$. Sections were incubated at 37 and $54^{\circ} \mathrm{C}$ for $24 \mathrm{~h}$ and $30 \mathrm{~min}$, respectively. Next, to remove the paraffin, slides were soaked in xylene and then rehydrated in a graded alcohol series. For antigen retrieval, the tissue sections were heated in EDTA (pH 9.0) using a microwave for $60 \mathrm{~min}$. Then, IHC was performed automatically using an Autostainer Plus ${ }^{\circledR}$ (Dako; Agilent Technologies, Inc., Santa Clara, CA, USA). Antibodies against 8-OHdG (dilution 1:200; cat. no. sc-66036; mouse monoclonal; Santa Cruz Biotechnology, Inc., Dallas, TX, USA) and the EnVision $+{ }^{\circledR}$ System (ready-to-use; Code K5007; Dako; Agilent Technologies, Inc.) were used as the primary and secondary antibodies, respectively. The slides were visualised using 3,3'-diaminobenzidine tetrahydrochloride and nuclei were counterstained with hematoxylin.

qIHC analysis of 8-OHdG levels. Immunostained tissue slides were digitised using a NanoZoomer 2.0HT digital slide scanner (Hamamatsu Photonics, Hamamatsu, Japan), and resulting whole-slide digital images in NDPI files were visualised using NDP.view2 software (Hamamatsu Photonics). NDPI files were converted to JPEG files using NDP.view2 software for the imaging analysis.

The proportion of positive 8-OHdG staining was estimated in the whole field of the slide by the pathologist. Furthermore, the intensity of cytoplasmic 8-OHdG staining was assessed using the average marker intensity, which was automatically calculated by the imaging analysis software (Tissue Studio ${ }^{\circledR} 4.0$; Definiens, München, Germany). Finally, the 8-OHdG value was calculated using the following calculation formula: Percentage of positive 8 - OHdG expression (\%) $\mathrm{x}$ average marker intensity.

Statistical analysis. Categorical variables are presented as numbers. The biomarkers for each background categorical variable are expressed as the median [interquartile range (IQR)], and Wilcoxon's signed-rank tests were used to compare biomarkers and background categorical variables. A Cox proportional hazards model was used in the univariate and multivariate analyses of disease-free survival (DFS) and disease-specific survival (DSS). Time-dependent receiver-operating characteristic (ROC) curves with Youden indices were used to identify the optimal cut-off values of 8-OHdG ratios (for both ELISA and qIHC), distinguishing the high- and low-risk groups with respect to the recurrence event. Kaplan-Meier curves of patients with high or low 8-OHdG ratios were plotted, and log-rank tests were conducted. All tests were two-tailed, and $\mathrm{P}<0.05$ was considered to indicate a statistically significant difference. All analyses were conducted using R Statistical Software (version 3.3.3; R Foundation for Statistical Computing, Vienna, Austria) and JMP Pro version 13 (SAS Institute, Inc., Cary, NC, USA). 
Table I. Relationship between clinicopathological characteristics and the 8-OHdG ratio.

\begin{tabular}{|c|c|c|c|c|c|}
\hline \multirow[b]{2}{*}{ Characteristics } & \multirow[b]{2}{*}{$\mathrm{n}$} & \multicolumn{2}{|c|}{ 8-OHdG ratio (ELISA) } & \multicolumn{2}{|c|}{ 8-OHdG ratio (qIHC) } \\
\hline & & Median (IQR) & $\begin{array}{c}\text { Univariate } \\
\text { P-value }\end{array}$ & Median (IQR) & $\begin{array}{c}\text { Univariate } \\
\text { P-value }\end{array}$ \\
\hline \multicolumn{6}{|l|}{ Age, years } \\
\hline$<75$ & 65 & $1.014(0.795-1.250)$ & \multirow[t]{2}{*}{0.0892} & $0.978(0.583-1.307)$ & \multirow[t]{2}{*}{0.5069} \\
\hline$\geq 75$ & 32 & $0.863(0.666-1.073)$ & & $1.138(0.432-1.838)$ & \\
\hline \multicolumn{6}{|l|}{ Sex } \\
\hline Male & 63 & $0.993(0.550-1.221)$ & \multirow[t]{2}{*}{0.3504} & $0.999(0.000-4.050)$ & \multirow[t]{2}{*}{0.1909} \\
\hline Female & 34 & $0.929(0.724-1.221)$ & & $0.949(0.000-3.558)$ & \\
\hline \multicolumn{6}{|l|}{ Tumor depth } \\
\hline$\leq \mathrm{T} 3$ & 69 & $0.934(0.778-1.182)$ & \multirow[t]{2}{*}{0.6021} & $1.047(0.587-1.486)$ & \multirow[t]{2}{*}{0.0587} \\
\hline $\mathrm{T} 4$ & 28 & $1.014(0.745-1.236)$ & & $0.791(0.440-1.041)$ & \\
\hline \multicolumn{6}{|c|}{ Lymph node metastasis } \\
\hline Absent & 54 & $0.883(0.675-1.114)$ & \multirow[t]{2}{*}{0.0265} & $1.013(0.550-1.515)$ & \multirow[t]{2}{*}{0.6680} \\
\hline Present & 43 & $1.031(0.825-1.303)$ & & $0.978(0.000-1.382)$ & \\
\hline \multicolumn{6}{|c|}{ Lymphatic invasion } \\
\hline Absent & 44 & $0.886(0.686-1.094)$ & \multirow[t]{2}{*}{0.0353} & $1.034(0.557-1.488)$ & \multirow[t]{2}{*}{0.6480} \\
\hline Present & 53 & $1.031(0.824-1.305)$ & & $0.978(0.566-1.324)$ & \\
\hline \multicolumn{6}{|l|}{ Venous invasion } \\
\hline Absent & 62 & $0.924(0.767-1.100)$ & \multirow[t]{2}{*}{0.1155} & $1.013(0.554-1.522)$ & \multirow[t]{2}{*}{0.4920} \\
\hline Present & 35 & $1.061(0.760-1.307)$ & & $0.978(0.561-1.329)$ & \\
\hline \multicolumn{6}{|c|}{ Adjuvant chemotherapy } \\
\hline Absent & 66 & $0.883(0675-1.146)$ & \multirow[t]{2}{*}{0.0189} & $1.034(0.522-1.484)$ & \multirow[t]{2}{*}{0.6340} \\
\hline Present & 31 & $1.050(0.934-1.285)$ & & $0.932(0.593-1.211)$ & \\
\hline
\end{tabular}

8-OHdG, 8-hydroxydeoxyguanosine; ELISA, enzyme-linked immunosorbent assay; qIHC, quantitative immunohistochemistry; IQR, interquartile range.

\section{Results}

Based on 8-OHdG ratio screening, 97 patients were finally enrolled in the study. The CONSORT flow diagram detailing the selection of patients is presented in Fig. 1. Five patients were deemed ineligible because adequate amounts of DNA for ELISA could not be extracted $(n=3)$ or the $8-\mathrm{OHdG}$ ratio for qIHC could not be calculated $(n=2)$.

Quantitative measurement of 8-OHdG levels via ELISA and qIHC. First, we quantitatively assessed $8-\mathrm{OHdG}$ in CRC tissue DNA using ELISA and examined 8-OHdG expression in CRC tissues via qIHC. According to ELISA, the median 8-OHdG level in normal tissue DNA was $1.176(\mathrm{IQR}=0.864-1.527)$ $\mathrm{ng} / \mathrm{ml}$, compared with 1.124 (IQR=0.792-1.577) $\mathrm{ng} / \mathrm{ml}$ in cancer tissue, with no significant difference identified (Fig. 2A). In IHC analysis, positive 8-OHdG staining was observed in the cytoplasm, but not in the nucleus. Fig. 2B shows examples of normal (Fig. 2B-a) and cancer tissue specimens (Fig. 2B-b, and -c) assessed via qIHC. The $8-\mathrm{OHdG}$ value in normal samples was estimated at 0.398 (Fig. 2B-a). The value in one CRC sample with strongly positive $8-\mathrm{OHdG}$ staining was estimated at 0.873 (Fig. 2B-b), whereas another CRC sample with weakly positive expression had a value of 0.131 (Fig. 2B-c). Fig. 2C shows that the median $8-\mathrm{OHdG}$ values in normal and CRC tissues were $0.280(\mathrm{IQR}=0.156-0.351)$ and $0.263(\mathrm{IQR}=0.116-0.412)$, respectively, with no significant difference observed.

Relationship between the 8-OHdG ratios in cancerous/normal tissues and clinicopathological characteristics of CRC. To eliminate the individual backgrounds of the 97 patients, we next determined $8-\mathrm{OHdG}$ ratios $(8-\mathrm{OHdG}$ level in cancerous tissue/8-OHdG level in normal tissue) using both ELISA and qIHC. Table I reveals the relationship between the clinicopathological characteristics and the $8-\mathrm{OHdG}$ ratios in DNA. No significant differences in the $8-\mathrm{OHdG}$ ratio in DNA were noted in terms of age ( $<75$ years vs. $\geq 75$ years), sex (male vs. female), tumor depth (T4 vs. $\leq \mathrm{T} 3$ ) or venous invasion. However, significant differences in the ratio were observed according to lymph node metastasis, lymphatic invasion and the use of postoperative adjuvant chemotherapy. Conversely, no clinicopathological factor was associated with a difference in the $8-\mathrm{OHdG}$ ratio in the cytoplasm (Table I). In addition, we performed correlation analysis between the $8-\mathrm{OHdG}$ ratio in DNA and the 8-OHdG ratio in the cytoplasm. The results revealed a negative linear correlation between the two 8-OHdG ratios; however, the coefficient was small $(\mathrm{r}=-0.229, \mathrm{P}=0.024)$ (data not shown). 


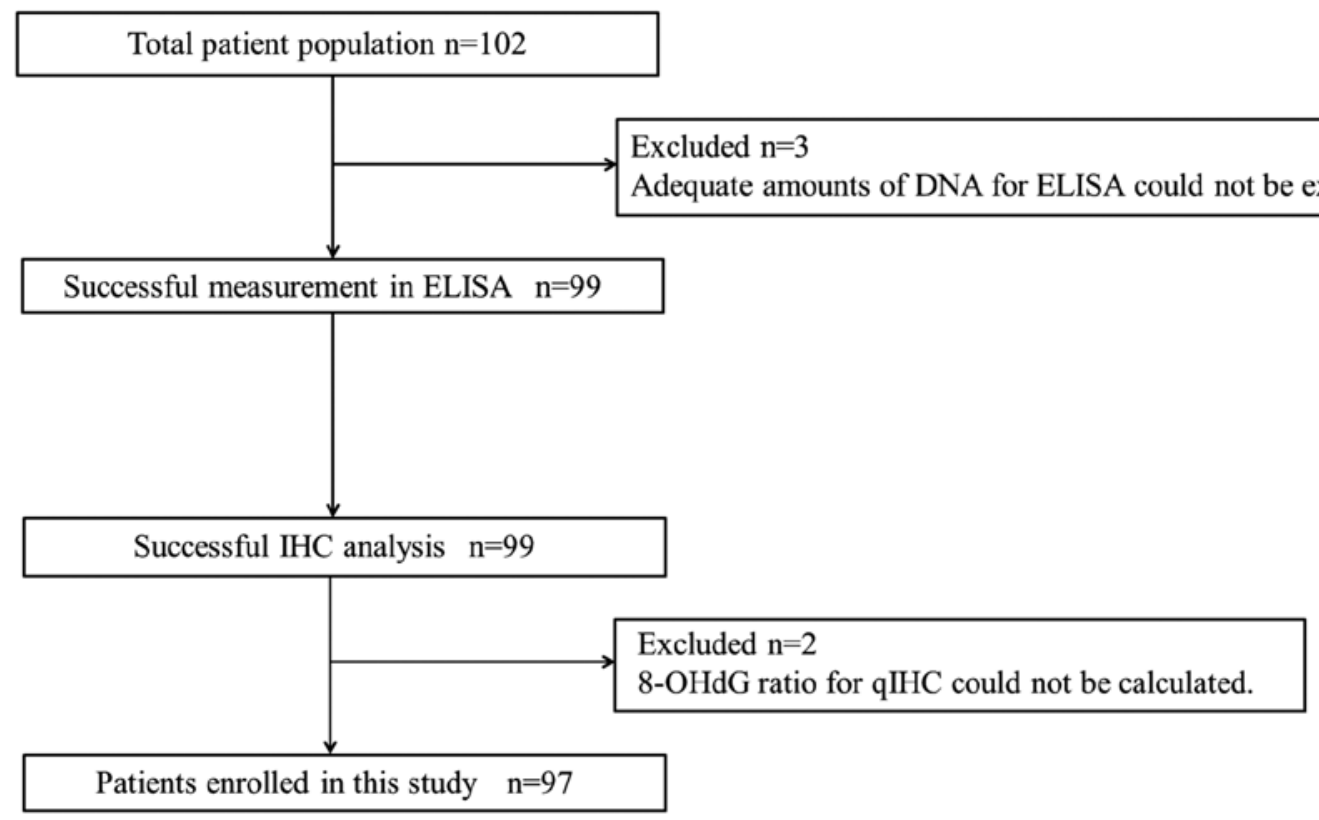

Figure 1. CONSORT flow diagram. CONSORT flow diagram detailing the selection of patients for analysis. Five tissue samples were not suitable for inclusion in the present study since adequate amounts of DNA for measurement using ELISA could not be extracted $(\mathrm{n}=3)$ or the 8-OHdG ratio for qIHC could not be calculated $(\mathrm{n}=2)$. ELISA, enzyme-linked immunosorbent assay; 8-OHdG, 8-hydroxydeoxyguanosine; qIHC, quantitative immunohistochemistry.
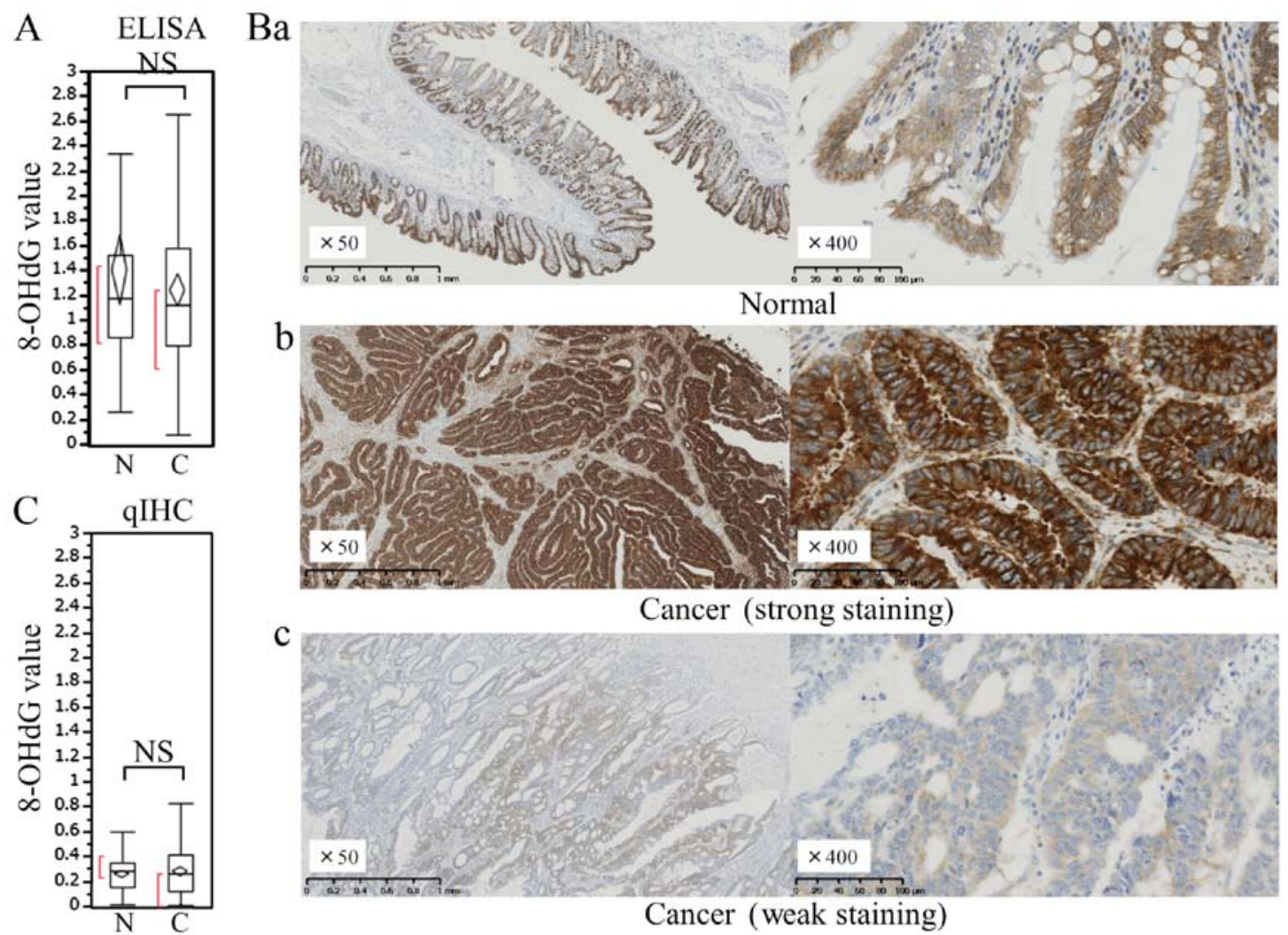

Cancer (strong staining)

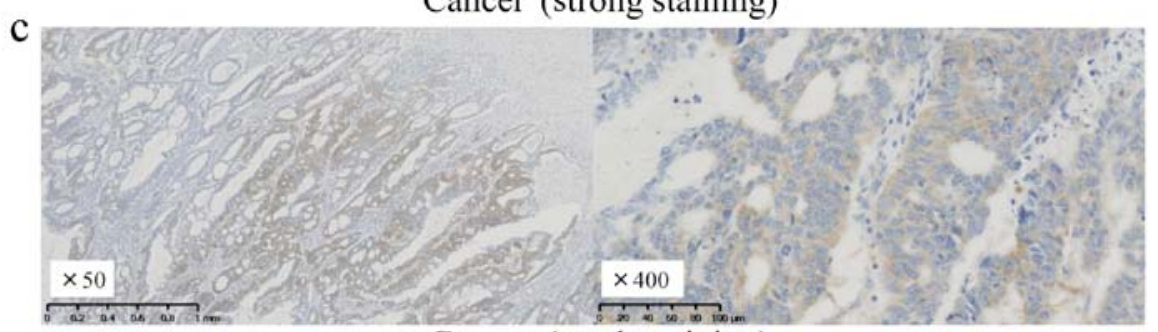

Cancer (weak staining)

Figure 2. 8-OHdG levels in CRC and normal tissues measured using ELISA or qIHC. (A) 8-OHdG levels in 97 pairs of normal and cancerous tissue DNA as estimated by ELISA. N, normal tissue DNA; C, cancer tissue DNA; NS, not significant. (B) Case presentation of 8-OHdG levels in normal and CRC tissues as determined by qIHC. a, Image of normal tissue with positive 8-OHdG staining in the cytoplasm. The proportion of positive 8-OHdG expression was $100 \%$. The mean staining intensity of $8-\mathrm{OHdG}$ in this area was automatically estimated as 0.398 using imaging analysis software. Thus, the 8-OHdG expression value for this tissue was 0.398 (100\% x 0.398). b, Image of cancer tissue with strong 8-OHdG staining in the cytoplasm. The proportion of positive 8-OHdG expression in this tissue was $100 \%$. The mean staining intensity of $8-\mathrm{OHdG}$ expression in this area was quantitatively measured as 0.873 . Thus, the $8-\mathrm{OHdG}$ expression value in this sample was $0.873(100 \%$ x 0.873$)$. c, Image of cancer tissue with weak 8-OHdG staining in the cytoplasm. The proportion of positive 8 -OHdG expression in this tissue was $60 \%$. The mean staining intensity of $8-\mathrm{OHdG}$ expression in this area was quantitatively measured as 0.218 . Thus, the 8-OHdG expression value in this sample was 0.131 (60\% x 0.218). (C) 8-OHdG levels in 97 pairs of normal and cancerous tissues as estimated by qIHC. $\mathrm{N}$, normal tissue; C, cancer tissue; NS, not significant; 8-OHdG, 8-hydroxydeoxyguanosine; CRC, colorectal cancer; ELISA, enzyme-linked immunosorbent assay; qIHC, quantitative immunohistochemistry. 
Table II. Univariate and multivariate analyses of disease-free survival using a Cox proportional hazard model.

\begin{tabular}{|c|c|c|c|c|}
\hline \multirow[b]{3}{*}{ Characteristics } & \multicolumn{4}{|c|}{ DFS } \\
\hline & \multicolumn{2}{|c|}{ Univariate analysis } & \multicolumn{2}{|c|}{ Multivariate analysis } \\
\hline & HR $(95 \% \mathrm{CI})$ & P-value & $\operatorname{HR}(95 \% \mathrm{CI})$ & P-value \\
\hline 8-OHdG (ELISA) & $5.15(2.59-10.23)$ & $<0.0001$ & $6.48(2.61-16.09)$ & 0.0001 \\
\hline 8-OHdG (qIHC) & $0.34(0.15-0.77)$ & 0.0094 & $0.14(0.04-0.51)$ & 0.0031 \\
\hline Age, years $(<75 / \geq 75)$ & $1.62(0.60-4.38)$ & 0.3453 & $0.85(0.25-2.96)$ & 0.8017 \\
\hline Sex (male/female) & $2.72(0.92-8.04)$ & 0.0705 & $0.30(0.09-0.96)$ & 0.0425 \\
\hline Tumor depth $(\leq \mathrm{T} 3 / \mathrm{T} 4)$ & $1.43(0.60-3.42)$ & 0.4160 & & \\
\hline Lymph node metastasis (+/-) & $2.42(1.02-5.77)$ & 0.0461 & & \\
\hline Lymphatic invasion (+/-) & $3.28(1.21-8.89)$ & 0.0197 & $2.62(0.87-7.93)$ & 0.0880 \\
\hline Venous invasion $(+/-)$ & $1.88(0.82-4.34)$ & 0.1384 & & \\
\hline Adjuvant chemotherapy (+/-) & $2.32(1.01-5.36)$ & 0.0484 & $2.44(0.97-6.12)$ & 0.0577 \\
\hline
\end{tabular}

DFS, disease-free survival; HR, hazard ratio; CI, confidence interval; 8-OHdG, 8-hydroxydeoxyguanosine; ELISA, enzyme-linked immunosorbent assay; qIHC, quantitative immunohistochemistry.

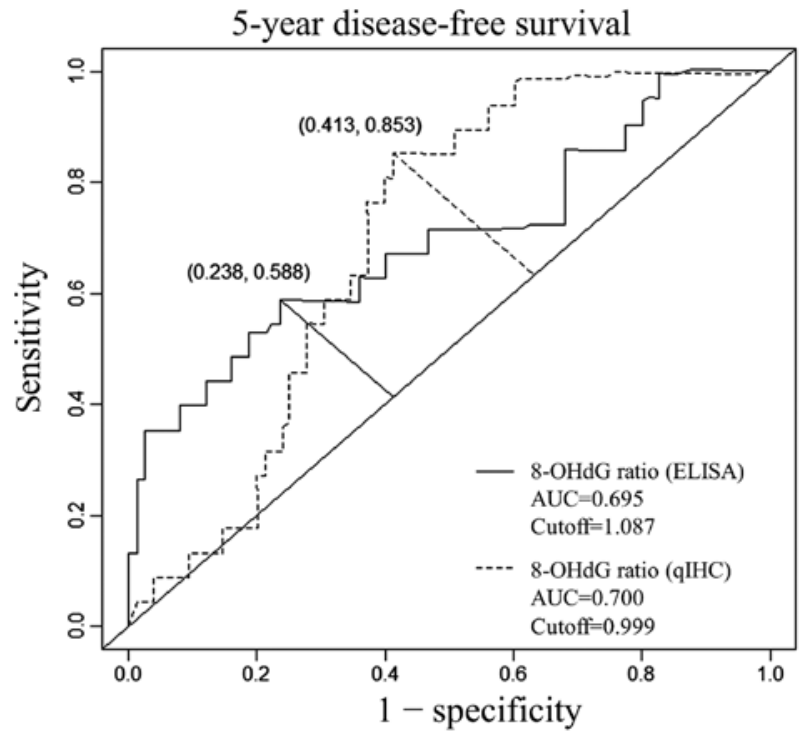

Figure 3. Time-dependent ROC curve. The ROC curve analysis indicated that the optimal cut-off values of the 8 -OHdG ratio were 1.087 by ELISA and 0.999 by qIHC. ROC, receiver-operating characteristic; 8-OHdG, 8-hydroxydeoxyguanosine; ELISA, enzyme-linked immunosorbent assay; qIHC, quantitative immunohistochemistry.

Relationship of 8-OHdG ratios with survival outcomes. During the observation period, 22 patients experienced CRC recurrence, which was fatal in 11 patients. We conducted analyses of DFS and DSS in the 97 enrolled patients using a Cox proportional hazard model (Tables II and III). Univariate analysis demonstrated that DFS was significantly correlated with the $8-\mathrm{OHdG}$ ratio in DNA [hazard ratio $(\mathrm{HR})=5.15 ; 95 \%$ confidence interval $(\mathrm{CI})=2.59-10.23$; $\mathrm{P}=0.0001]$, the $8-\mathrm{OHdG}$ ratio in the cytoplasm $(\mathrm{HR}=0.34$; 95\% $\mathrm{CI}=0.15-0.77 ; \mathrm{P}=0.0094)$, ly mph node metastasis $(\mathrm{HR}=2.42 ; 95 \% \mathrm{CI}=1.02-5.77 ; \mathrm{P}=0.0461)$, lymphatic invasion $(\mathrm{HR}=3.28 ; 95 \% \mathrm{CI}, 1.21-8.89 ; \mathrm{P}=0.0197)$ and the receipt of postoperative adjuvant chemotherapy $(\mathrm{HR}=2.32 ; 95 \% \mathrm{CI}$, 1.01-5.36; $\mathrm{P}=0.0484$ ) (Table II). Additionally, the 8-OHdG ratio in DNA was significantly correlated with $\mathrm{DSS}(\mathrm{HR}=8.14$; 95\% CI, 3.51-18.90; $\mathrm{P}<0.0001$ ) (Table III). According to multivariate analyses, the $8-\mathrm{OHdG}$ ratio in DNA $(\mathrm{HR}=6.48$; 95\% CI, 2.61-16.09; $\mathrm{P}=0.0001)$, the $8-\mathrm{OHdG}$ ratio in the cytoplasm $(\mathrm{HR}=0.14 ; 95 \% \mathrm{CI}, 0.04-0.51 ; \mathrm{P}=0.0031)$ and sex ( $\mathrm{HR}=0.30 ; 95 \% \mathrm{CI}, 0.09-0.96 ; \mathrm{P}=0.0425)$ were independently correlated with DFS (Table II). Furthermore, the 8-OHdG ratio in both DNA $(\mathrm{HR}=10.74 ; 95 \% \mathrm{CI}, 3.54-32.6 ; \mathrm{P}<0.0001)$ and the cytoplasm $(\mathrm{HR}=0.17 ; 95 \% \mathrm{CI}, 0.04-0.83 ; \mathrm{P}=0.0285)$ were independently correlated with DSS (Table III).

ROC curve analysis and cut-off value for predicting CRC recurrence. Time-dependent ROC curve analysis was conducted to determine the most informative cut-off point of the 8-OHdG ratio in both DNA and the cytoplasm for predicting CRC recurrence. Fig. 3 reveals the estimated ROC curve with respect to the 5-year DFS. The ROC curve analysis indicated that the optimal cut-off $8-\mathrm{OHdG}$ ratio in DNA, as determined using the Youden index, was 1.087 (sensitivity $=58.8 \%$; specificity $=76.2 \%$ ). The optimal cut-off value for the $8-\mathrm{OHdG}$ ratio in the cytoplasm was 0.999 (sensitivity $=85.3 \%$; specificity $=58.7 \%$ ) (Fig. 3).

Survival curves according to the 8-OHdG ratios estimated using both ELISA and qIHC. Based on ROC analyses, the impact of the 8-OHdG ratio in DNA or the cytoplasm on DFS and DSS was further analysed using Kaplan-Meier curves (Fig. 4). DFS and DSS were significantly poorer in the high 8-OHdG ratio in DNA group than in the low ratio group (Fig. 4A; $\mathrm{P}=0.0043$ for DFS, $\mathrm{P}=0.0044$ for DSS). By contrast, DFS was significantly poorer in the low $8-\mathrm{OHdG}$ ratio in the cytoplasm group than in the high ratio group, whereas DSS was not significantly different between these groups (Fig. 4B; $\mathrm{P}=0.0006$ for DFS, $\mathrm{P}=0.0520$ for DSS). Furthermore, we divided the patients into three groups as follows. Group I 
Table III. Univariate and multivariate analyses of disease-specific survival using a Cox proportional hazard model.

\begin{tabular}{|c|c|c|c|c|}
\hline \multirow[b]{3}{*}{ Characteristics } & \multicolumn{4}{|c|}{ DSS } \\
\hline & \multicolumn{2}{|c|}{ Univariate analysis } & \multicolumn{2}{|c|}{ Multivariate analysis } \\
\hline & $\mathrm{HR}(95 \% \mathrm{CI})$ & P-value & HR $(95 \% \mathrm{CI})$ & P-value \\
\hline 8-OHdG (ELISA) & $8.14(3.51-18.90)$ & $<0.0001$ & $10.74(3.54-32.60)$ & $<0.0001$ \\
\hline 8-OHdG (qIHC) & $0.32(0.09-1.06)$ & 0.0618 & $0.17(0.04-0.83)$ & 0.0285 \\
\hline Age, years $(<75 / \geq 75)$ & $0.72(0.21-2.45)$ & 0.5953 & $2.03(0.44-9.25)$ & 0.3618 \\
\hline Sex (male/female) & $5.61(0.72-43.79)$ & 0.1003 & $0.16(0.02-1.29)$ & 0.0845 \\
\hline Tumor depth $(\leq \mathrm{T} 3 / \mathrm{T} 4)$ & $3.09(0.94-10.13)$ & 0.0623 & & \\
\hline Lymph node metastasis (+/-) & $2.24(0.66-7.66)$ & 0.1978 & & \\
\hline Lymphatic invasion (+/-) & $2.40(0.64-9.04)$ & 0.1968 & & \\
\hline Venous invasion $(+/-)$ & $1.52(0.46-4.99)$ & 0.4869 & & \\
\hline Adjuvant chemotherapy (+/-) & $1.13(0.33-3.87)$ & 0.8418 & & \\
\hline
\end{tabular}

DSS, disease-specific survival; HR, hazard ratio; CI, confidence interval; 8-OHdG, 8-hydroxydeoxyguanosine; ELISA, enzyme-linked immunosorbent assay; qIHC, quantitative immunohistochemistry.
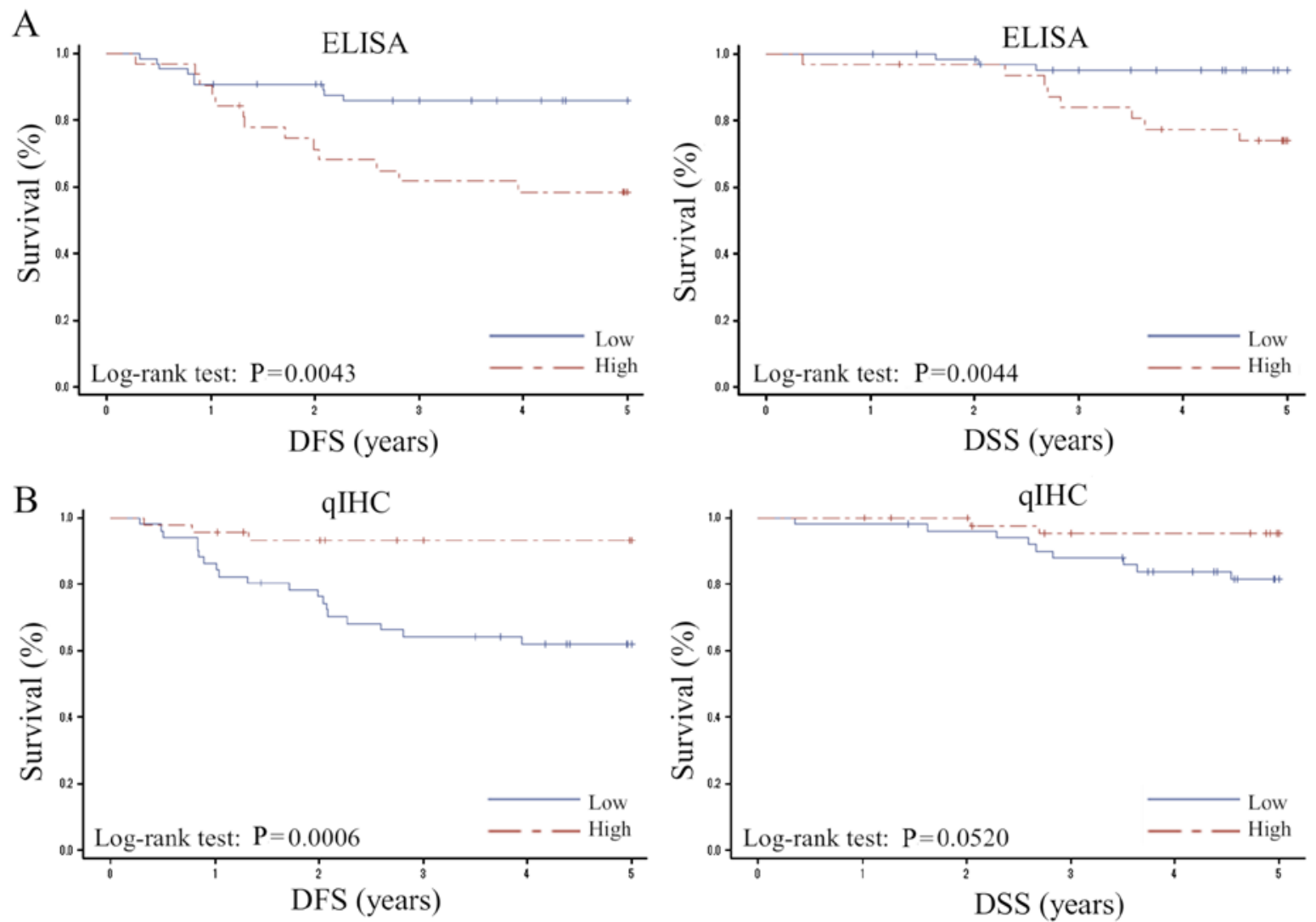

Figure 4. Kaplan-Meier curves of DFS and DSS according to the 8-OHdG ratios estimated using ELISA or qIHC. (A) DFS or DSS for patient groups with high or low 8-OHdG ratios using ELISA. High 8-OHdG ratio (ELISA) group, $\geq 1.087$; low 8-OHdG ratio (ELISA) group, $<1.087$. (B) DFS or DSS for patient groups with high or low 8-OHdG ratios using qIHC. High 8-OHdG ratio group, $\geq 0.999$; low 8-OHdG ratio group, $<0.999$. DFS, disease-free survival; DSS, disease-specific survival; 8-OHdG, 8-hydroxydeoxyguanosine; ELISA, enzyme-linked immunosorbent assay; qIHC, quantitative immunohistochemistry.

included patients with an $8-\mathrm{OHdG}$ ratio in DNA $\geq 1.087$ and an 8 -OHdG ratio in the cytoplasm $<0.999$ [ $n=33(34.0 \%)]$. Group II included patients with an $8-\mathrm{OHdG}$ ratio in DNA $<1.087$ and an 8 -OHdG ratio in the cytoplasm $\geq 0.999$ [ $n=19$ (19.6\%)].
Group III included patients with an $8-\mathrm{OHdG}$ ratio in DNA $\geq 1.087$ and an $8-\mathrm{OHdG}$ ratio in the cytoplasm $\geq 0.999$, or an $8-\mathrm{OHdG}$ ratio in DNA $<1.087$ and an $8-\mathrm{OHdG}$ ratio in the cytoplasm $<0.999[\mathrm{n}=45(46.4 \%)]$. Fig. 5 demonstrated that 

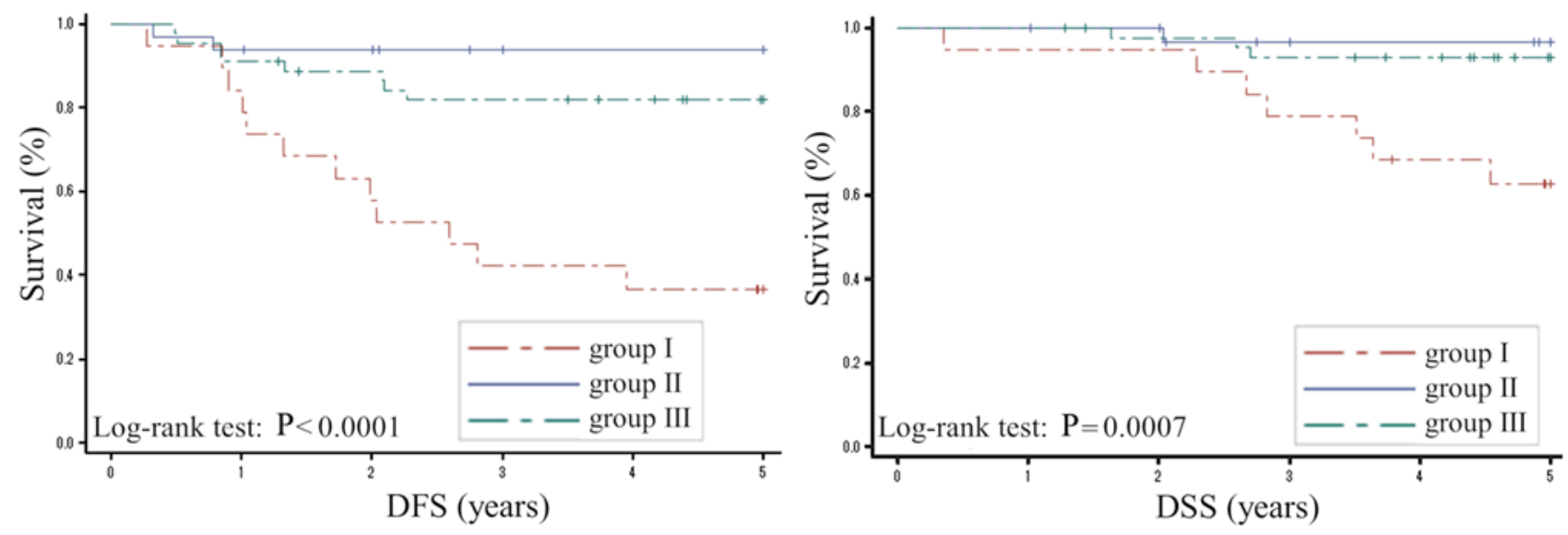

Figure 5. Kaplan-Meier curves for DFS and DSS according to 8-OHdG ratios estimated by the combination of ELISA and qIHC. DFS or DSS in patients divided into three groups: Group I, high 8-OHdG ratio (ELISA) and low 8-OHdG ratio (qIHC); group II, low 8-OHdG ratio (ELISA) and high 8-OHdG ratio (qIHC); group III, others. DFS, disease-free survival; DSS, disease-specific survival; 8-OHdG, 8-hydroxydeoxyguanosine; ELISA, enzyme-linked immunosorbent assay; qIHC, quantitative immunohistochemistry.

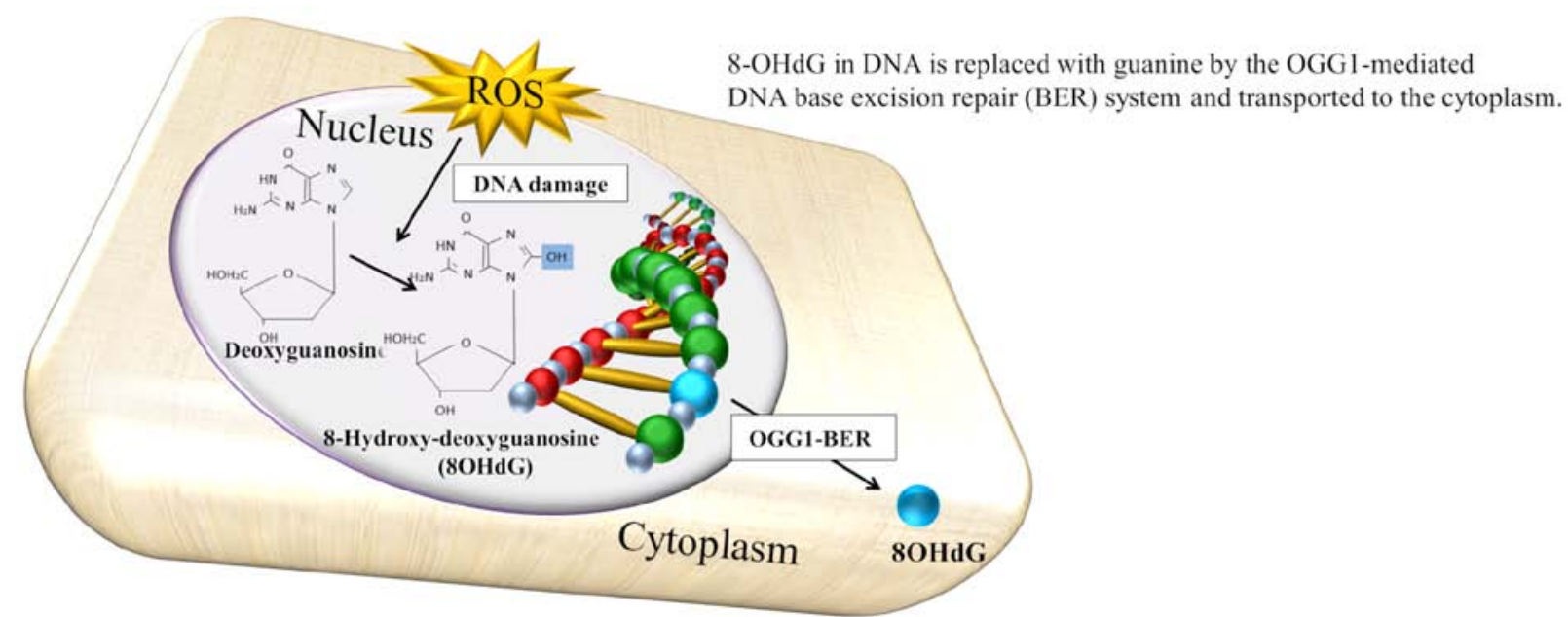

Accumulation of DNA damage (Group I)

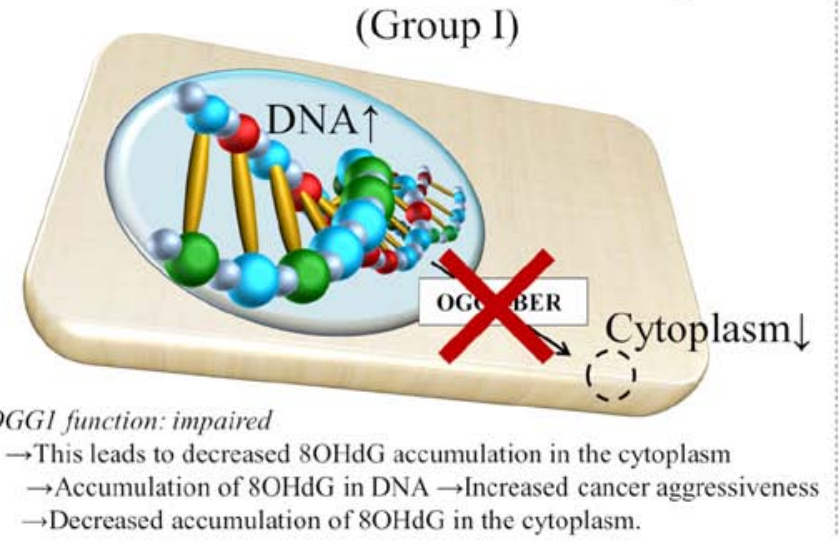

Repair of DNA damage (Group II)

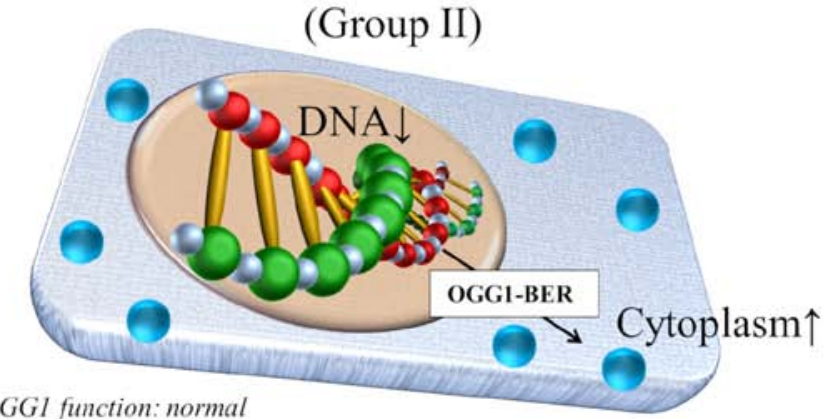

OGGl function: normal

$\rightarrow$ DNA damage is repaired, and $8 \mathrm{OHdG}$ is transported to the cytoplasm

$\rightarrow$ Accumulation of $8 \mathrm{OHdG}$ in the cytoplasm

$\rightarrow$ Decreased accumulation of $8 \mathrm{OHdG}$ in DNA

Figure 6. Relationship between 8-OHdG levels in DNA and the cytoplasm and its potential regulation by OGG1 function. 8-OHdG in DNA is replaced with guanine by the OGG1-mediated DNA base excision repair system and transported to the cytoplasm. OGG1 function, impaired. 8-OHdG in DNA is not excised, resulting in low 8-OHdG levels in cytoplasm. Accumulation of 8-OHdG in DNA increases cancer aggressiveness (group I in our study). OGG1 function, normal. 8-OHdG in DNA is repaired by the OGG-1-mediated base excision repair system. Thereafter the excised 8-OHdG mononucleotides are transported to the cytoplasm, leading to high 8-OHdG levels in the cytoplasm and low 8-OHdG levels in DNA, thereby suppressing cancer aggressiveness (group II in our study). 8-OHdG, 8-hydroxydeoxyguanosine; OGG1, 8-OHdG DNA glycosylase.

the DFS and DSS were markedly lower in group I than in the other groups $(\mathrm{P}<0.0001$ for DFS, $\mathrm{P}=0.0007$ for DSS). Finally, Table IV presents the comparative analysis of the hazard ratios for recurrence or death among groups I-III. Group I had an increased risk of recurrence or death compared with the findings in the other groups (Table IV). 
Table IV. Comparison of the risk of recurrence or death among groups I-III.

\begin{tabular}{|c|c|c|c|c|}
\hline \multirow[b]{2}{*}{ Categorical variable } & \multicolumn{2}{|c|}{ Recurrence } & \multicolumn{2}{|c|}{ Death } \\
\hline & $\mathrm{HR}(95 \% \mathrm{CI})$ & P-value & $\mathrm{HR}(95 \% \mathrm{CI})$ & P-value \\
\hline Group I & 1 & - & 1 & - \\
\hline Group II & $0.08(0.02-0.34)$ & 0.00076 & $0.08(0.01-0.65)$ & 0.01792 \\
\hline Group III & $0.23(0.09-0.56)$ & 0.00133 & $0.17(0.04-0.66)$ & 0.01044 \\
\hline
\end{tabular}

HR, hazard ratio; CI, confidence interval.

\section{Discussion}

8-OHdG levels have been previously measured in serum, leukocytes and urine to assess oxidative stress in humans $(4,18)$. Studies have also investigated plasma 8-OHdG levels in patients with CRC and evaluated their correlations with clinicopathological variables or patient survival $(19,20)$. However, no study has identified significant correlations of plasma 8-OHdG levels with such factors $(19,20)$. A recent study also analysed 8-OHdG levels in CRC tumors via high-performance liquid chromatography (21). The results illustrated that $8-\mathrm{OHdG}$ levels were higher in cancerous tissues than in the corresponding normal tissues, whereas no significant relationship was observed between 8-OHdG levels and tumor stage (21).

In the present study, 8-OHdG levels in CRC tissues were quantitatively assessed via ELISA and qIHC. First, 8-OHdG levels in tissue DNA were measured by ELISA using both cancerous and corresponding normal tissues. The results indicated that 8-OHdG levels in DNA were not significantly different between normal and cancerous tissues. Next, 8-OHdG levels in the cytoplasm were also investigated via qIHC using imaging analysis software. Positive $8-\mathrm{OHdG}$ staining was observed in the cytoplasm, but not in the nucleus, in both cancerous and normal tissues. In line with the ELISA observations, 8-OHdG levels in the cytoplasm were not significantly different between normal and cancerous tissues.

Previous studies reported that ROS accumulation in humans varies among individuals because ROS generation can be influenced by ageing $(22,23)$, smoking $(24)$ and chronic diseases such as diabetes (25). Additionally, the intestinal epithelium is directly exposed to red meat, fat and alcohol, thereby exogenously increasing ROS generation $(26,27)$. Therefore, to eliminate such backgrounds, we estimated the 8-OHdG ratio between cancerous and normal tissues using both ELISA and qIHC and determined the 8-OHdG ratios as the cancer-dependent accumulation of 8-OHdG. To the best of our knowledge, only one study has evaluated the relationship between the outcomes of patients with cancer and 8-OHdG ratios in DNA from ovarian cancer tissue (18), revealing that survival was worse in patients with higher 8 -OHdG ratios than in those with lower 8-OHdG ratios (18). Based on these concepts, we examined the correlation of the 8 -OHdG ratio with several clinicopathological factors and patient survival. The 8-OHdG ratio in DNA was significantly correlated with lymph node metastasis, lymphatic invasion and a history of adjuvant chemotherapy. The results suggested that the cancer-dependent accumulation of 8-OHdG in DNA accelerates tumor progression in CRC. G:C to T:A mutations caused by $8-\mathrm{OHdG}$ accumulation may activate genes related to cancer invasion or metastasis. Alternatively, since 8-OHdG accumulation in DNA results from ROS-induced oxidative damage in DNA, excessive ROS generation may increase CRC invasion and metastasis by directly activating signal transduction molecules in patients with high $8-\mathrm{OHdG}$ ratios in DNA (2). Contrarily, the 8-OHdG ratio in cytoplasm had no statistical correlation with any clinicopathologic factors.

Regarding survival, multivariate analysis revealed that high 8-OHdG ratios in DNA were independently correlated with poor DFS and DSS. Notably, low 8-OHdG ratios in the cytoplasm were also significantly correlated with poor DFS and DSS. Previously, an IHC study using breast cancer tissues demonstrated that DSS was significantly poorer among patients with negative 8-OHdG than in those with positive expression (28). This study may support our qIHC findings, although the breast cancer study did not assess 8-OHdG levels quantitatively (28). Using Kaplan-Meier curves, we further revealed that the co-existence of a high 8-OHdG ratio in DNA and a low 8-OHdG ratio in the cytoplasm was associated with significantly poorer DFS and DSS than other combinations of these variables.

In the present study, we did not address the mechanism by which the 8-OHdG ratio in DNA or the cytoplasm inversely affected the surgical outcomes of patients with CRC. Concerning this point, a possible mechanism involved 8-OHdG DNA glycosylase (OGG1), a base excision repair enzyme that participates in DNA repair $(16,29)$. To avoid DNA mutation, OGG1 removes 8-OHdG residues opposite cytosine from DNA, and the excised 8-OHdG mononucleotides can be transported to the cytoplasm $(16,29)$. Therefore, our interpretation is that 8-OHdG localisation in nuclear DNA or the cytoplasm may be determined by OGG1 activity in CRC. When OGG1 functions normally, incorporated $8-\mathrm{OHdG}$ is effectively excised from DNA, and high amounts of 8-OHdG mononucleotides may be effluxed to the cytoplasm. By contrast, if OGG1 activity is impaired, then significant 8-OHdG accumulation can occur in nuclear DNA, resulting in G:C to T:A mutations and leading to CRC progression and poor patient prognoses. Under this situation, 8-OHdG mononucleotide levels in the cytoplasm may be decreased, resulting in the detection of low levels via qIHC. Fig. 6 illustrates a possible mechanism by which 8 -OHdG may be accumulated in nuclear DNA owing to impaired OGG1 activity, thereby leading to increasing CRC aggressiveness. Additionally, we could not detect $8-\mathrm{OHdG}$ expression 
in nuclear DNA by IHC. Higher-order chromatin structures may disturb the immunological detection of $8-\mathrm{OHdG}$ by the antibody used in the present study. With respect to differences in OGG1 activity in cancers, several studies linked the OGG1 Ser326Cys polymorphism to OGG1 activity $(30,31)$. In CRC, a previous study analysed the frequencies of the Ser326Ser, Ser326Cys and Cys326Cys genotypes in CRC tissues; however, the literature did not report a significant correlation between any of the three polymorphic patterns and OGG1 activity (32). In the future, further research evaluating the relationships among OGG1 polymorphisms, OGG1 activity and 8-OHdG ratios may offer new insights into the pathogenesis of 8-OHdG-mediated CRC progression.

In conclusion, the present study demonstrated that cancer-dependent $8-\mathrm{OHdG}$ accumulation in tissue DNA, which was determined as the ratio of $8-\mathrm{OHdG}$ levels between cancerous and normal tissues via ELISA, may be an important factor for assessing ROS-mediated oxidative stress in CRC tissue DNA. Furthermore, assessing $8-\mathrm{OHdG}$ ratios using the combination of ELISA and qIHC could represent a powerful tool for predicting cancer recurrence and post-operative survival among patients with CRC.

\section{Acknowledgements}

We would like to thank Joe Barber Jr, PhD, from Edanz Group (www.edanzediting.com/ac) for editing a draft of this manuscript.

\section{Funding}

The present study was supported by JSPS KAKENHI Grants-in-Aid for Scientific Research (Research Project no. $18 \mathrm{~K} 08650)$.

\section{Availability of data and materials}

Individual-level data may be shared on request if all legal and ethical requirements are met. Requests should be sent to the corresponding author.

\section{Authors' contributions}

YKi and HK conceived and designed the experiments. HK and $\mathrm{KK}$ performed laboratory work and planning of the sequencing. HK performed all bioinformatical analyses. TT TM and $\mathrm{HN}$ has assisted with the planning of the work and have also performed laboratory work. HK, KK, YKo and TM have collected all the samples. HK and SK performed all statistical analyses. HK, YKo and HN wrote the study. All authors discussed the results and commented on the manuscript. All authors read and approved the manuscript and agree to be accountable for all aspects of the research in ensuring that the accuracy or integrity of any part of the work are appropriately investigated and resolved.

\section{Ethics approval and consent to participate}

The present study was approved by the Saga University Institutional Review Board (Saga, Japan; permission no. 2018-01-R-04). Each enrolled patient signed written informed consent prior to sample collection.

\section{Patient consent for publication}

Not applicable.

\section{Competing interests}

The authors declare no competing financial or non-financial interests.

\section{References}

1. Boring CC, Squires TS and Tong T: Cancer statistics, 1993. CA Cancer J Clin 43: 7-26, 1993.

2. Prasad S, Gupta SC and Tyagi AK: Reactive oxygen species (ROS) and cancer: Role of antioxidative nutraceuticals. Cancer Lett 387: 95-105, 2017.

3. Acharya A, Das I, Chandhok D and Saha T: Redox regulation in cancer: A double-edged sword with therapeutic potential. Oxid Med Cell Longev 3: 23-34, 2010.

4. Yang W, Zou L, Huang C and Lei Y: Redox regulation of cancer metastasis: Molecular signaling and therapeutic opportunities. Drug Dev Res 75: 331-341, 2014.

5. Tochhawng L, Deng S, Pervaiz S and Yap CT: Redox regulation of cancer cell migration and invasion. Mitochondrion 13: 246-253, 2013.

6. Sena LA and Chandel NS: Physiological roles of mitochondrial reactive oxygen species. Mol Cell 48: 158-167, 2012.

7. Tanaka T, Kitajima Y, Miyake S, Yanagihara K, Hara H, Nishijima-Matsunobu A, Baba K, Shida M, Wakiyama K, Nakamura J, et al: The apoptotic effect of HIF-1 $\alpha$ inhibition combined with glucose plus insulin treatment on gastric cancer under hypoxic conditions. PLoS One 10: e0137257, 2015.

8. Kamino H, Nakamura Y, Tsuneki M, Sano H, Miyamoto Y, Kitamura N, Futamura M, Kanai Y, Taniguchi H, Shida D, et al: Mieap-regulated mitochondrial quality control is frequently inactiveated in human colorectal cancer. OncogenesisJan 5: e181, 2016.

9. Levi F, Pasche C, La Vecchia C, Lucchini F and Franceschi S: Food groups and colorectal cancer risk. Br J Cancer 79: 1283-1287, 1999

10. Azeem S, Gillani SW, Siddiqui A, Jandrajupalli SB, Poh V and Syed Sulaiman SA: Diet and colorectal cancer risk in Asia - a systemic review. Asian Pac J Cancer Prev 16: 5389-5396, 2015.

11. Ray PD, Huang BW and Tsuji Y: Reactive oxygen species (ROS) homeostasis and redox regulation in cellular signaling. Cell Signal 24: 981-990, 2012.

12. Kryston TB, Georgiev AB, Pissis P and Georgakilas AG: Role of oxidative stress and DNA damage in human carcinogenesis. Mutat Res 711: 193-201, 2011.

13. Lin X, Zheng W, Liu J, Zhang Y, Qin H, Wu H, Xue B, Lu Y and Shen P: Oxidative stress in malignant melanoma enhances tumor necrosis factor- $\alpha$ secretion of tumor-associated macrophages that promote cancer cell invasion. Antioxid Redox Signal 19: 1337-1355, 2013.

14. Wartenberg M, Budde P, De Mareés M, Grünheck F, Tsang SY, Huang Y, Chen ZY, Hescheler J and Sauer H: Inhibition of tumor-induced angiogenesis and matrix-metalloproteinase expression in confrontation cultures of embryoid bodies and tumor spheroids by plant ingredients used in traditional chinese medicine. Lab Invest 83: 87-98, 2003.

15. Valko M, Izakovic M, Mazur M, Rhodes CJ and Telser J: Role of oxygen radicals in DNA damage and cancer incidence. Mol Cell Biochem 266: 37-56, 2004.

16. Nakabeppu Y: Cellular levels of 8-oxoguanine in either DNA or the nucleotide pool play pivotal roles in carcinogenesis and survival of cancer cells. Int J Mol Sci 15: 12543-12557, 2014.

17. Wu LL, Chiou CC, Chang PY and Wu JT: Urinary 8-OHdG: A marker of oxidative stress to DNA and a risk factor for cancer, atherosclerosis and diabetics. Clin Chim Acta 339: 1-9, 2004.

18. Xu X, Wang Y, Guo W, Zhou Y, Lv C, Chen X and Liu K: The significance of the alteration of $8-\mathrm{OHdG}$ in serous ovarian carcinoma. J Ovarian Res 6: 74, 2013. 
19. Sato T, Takeda H, Otake S, Yokozawa J, Nishise S, Fujishima S, Orii T, Fukui T, Takano J, Sasaki Y, et al: Increased plasma levels of 8-hydroxydeoxyguanosine are associated with development of colorectal tumors. J Clin Biochem Nutr 47: 59-63, 2010.

20. Dincer Y, Himmetoglu S, Akcay T, Ersoy EY, Gunes KN and Tortum O: Prognostic significances of oxidative DNA damage evaluated by 8 -hydroxy-deoxyguanosine and antioxidant enzymes in patients undergoing resection of gastric and colon carcinoma. Neoplasma 54: 131-136, 2007.

21. Płachetka A, Adamek B, Strzelczyk JK, Krakowczyk $Ł$, Migula P, Nowak P and Wiczkowski A: 8-hydroxy-2'-deoxyguanosine in colorectal adenocarcinoma--is it a result of oxidative stress? Med Sci Monit 19: 690-695, 2013.

22. Kaneko T, Tahara S and Matsuo M: Non-linear accumulation of 8-hydroxy-2'-deoxyguanosine, a marker of oxidized DNA damage, during aging. Mutat Res 316: 277-285, 1996.

23. Shigenaga MK, Hagen TM and Ames BN: Oxidative damage and mitochondrial decay in aging. Proc Natl Acad Sci USA 91: 10771-10778, 1994.

24. Leanderson P and Tagesson C: Cigarette smoke-induced DNA damage in cultured human lung cells: Role of hydroxyl radicals and endonuclease activation. Chem Biol Interact 81: 197-208, 1992

25. Nishikawa T, Sasahara T, Kiritoshi S, Sonoda K, Senokuchi T, Matsuo T, Kukidome D, Wake N, Matsumura T, Miyamura N, et al Evaluation of urinary 8-hydroxydeoxy-guanosine as a novel biomarker of macrovascular complications in type 2 diabetes. Diabetes Care 26: 1507-1512, 2003.
26. Glei M, Latunde-Dada GO, Klinder A, Becker TW, Hermann U, Voigt $\mathrm{K}$ and Pool-Zobel BL: Iron-overload induces oxidative DNA damage in the human colon carcinoma cell line HT29 clone 19A. Mutat Res 519: 151-161, 2002.

27. Fearon ER and Vogelstein B: A genetic model for colorectal tumorigenesis. Cell 61: 759-767, 1990.

28. Sova H, Jukkola-Vuorinen A, Puistola U, Kauppila S and Karihtala P: 8-Hydroxydeoxyguanosine: A new potential independent prognostic factor in breast cancer. $\mathrm{Br} \mathrm{J}$ Cancer 102: 1018-1023, 2010.

29. Boiteux S and Radicella JP: Base excision repair of 8-hydroxyguanine protects DNA from endogenous oxidative stress. Biochimie 81: 59-67, 1999.

30. Gackowski D, Speina E, Zielinska M, Kowalewski J, Rozalski R, Siomek A, Paciorek T, Tudek B and Olinski R: Products of oxidative DNA damage and repair as possible biomarkers of susceptibility to lung cancer. Cancer Res 63: 4899-4902, 2003.

31. Paz-Elizur T, Ben-Yosef R, Elinger D, Vexler A, Krupsky M, Berrebi A, Shani A, Schechtman E, Freedman L and Livneh Z: Reduced repair of the oxidative 8-oxoguanine DNA damage and risk of head and neck cancer. Cancer Res 66: 11683-11689, 2006.

32. Kondo S, Toyokuni S, Tanaka T, Hiai H, Onodera H, Kasai H and Imamura M: Overexpression of the hOGG1 gene and high 8-hydroxy-2'-deoxyguanosine (8-OHdG) lyase activity in human colorectal carcinoma: Regulation mechanism of the $8-\mathrm{OHdG}$ level in DNA. Clin Cancer Res 6: 1394-1400, 2000. 